\title{
Positive results for anifrolumab in phase III SLE trial
}

The TULIP-2 trial of anifrolumab,
the phase III
trials provide
encouraging
evidence of
interferon receptor (IFNAR), in
active systemic lupus erythematosus
of anifrolumab
(SLE) has met its primary end point.
"This is the first successful phase III
trial in SLE since the belimumab
program 10 years ago, highlights
study investigator Eric Morand.
The results revive hopes that the
drug will be approved for use in SLE,
following the failure of the earlier
TULIP-1 phase III study to meet its
primary end point.
In TULIP-2, a BILAG-based
Composite Lupus Assessment
(BICLA) response occurred in 86 of
180 patients (47.8\%) who received
anifrolumab at week 52, compared
with 57 of 182 (31.5\%) of those who
received placebo. "The improvement
in BICLA - a measure that requires

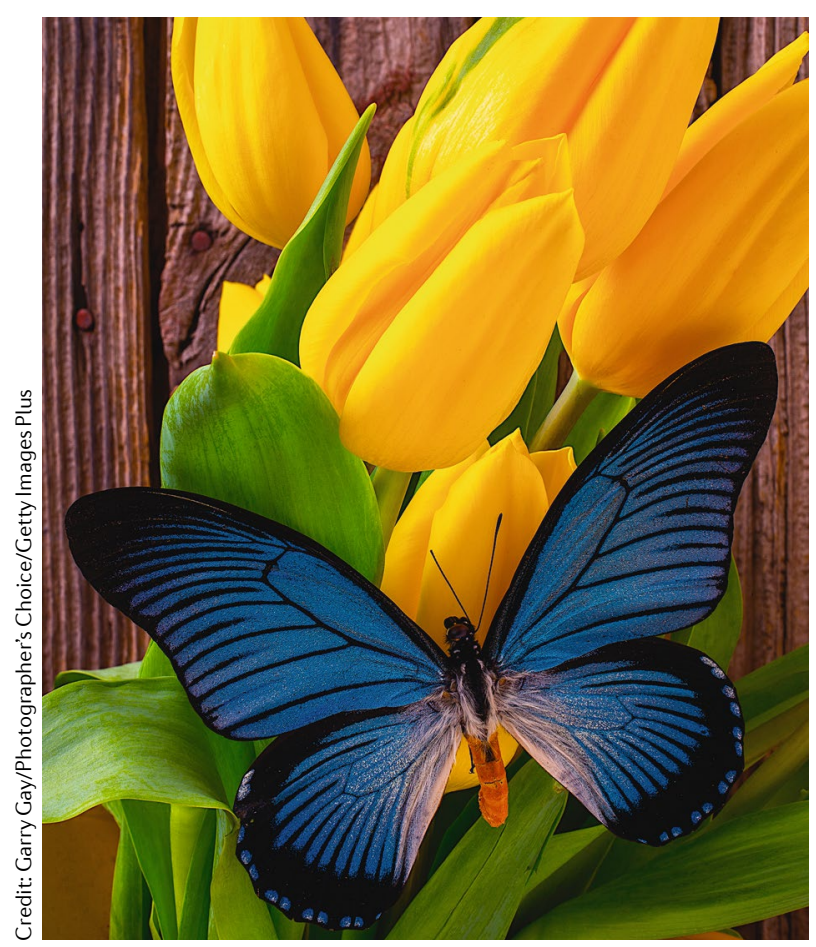

improvement in all severe or moderately severe organ system manifestations - occurred quite early after the initiation of treatment and was sustained throughout the 52-week study," notes SLE researcher Mary Crow, who was not involved in the study.

Type I interferon has long been implicated in the pathogenesis of SLE. "More than 20 years of basic science and translational research identifying the type I interferon pathway as prominently expressed in patients with SLE created a very robust case for scientific testing of the interferon hypothesis in SLE," says Morand. By antagonizing IFNAR, the common interferon receptor subunit, anifrolumab prevents signalling by all type I interferon subtypes.

Although the TULIP-1 trial of anifrolumab failed to meet its primary end point, the SLE responder index (SRI) 4 composite measure, the drug showed efficacy in some pre-specified secondary end points, including BICLA response. Following a careful analysis of the TULIP-1 data (but before the unblinding of TULIP-2 trial data), the primary outcome measure for TULIP-2 was changed from SRI4 to BICLA in a protocol amendment. "In contrast to BICLA, SRI4 reflects presence of disease activity related to a lupus manifestation rather than severity of a manifestation, so use of BICLA allows for assessment of incremental response rather than requiring more complete resolution of a disease manifestation," Crow explains. Notably, SRI4 seemed to improve more with anifrolumab than with placebo in TULIP-2, although this outcome was not formally assessed for statistical significance.
Other secondary end points met in TULIP-2 included reductions in the glucocorticoid dose and in the severity of skin disease in patients treated with anifrolumab as compared with placebo. However, no differences between the groups were seen in counts of swollen or tender joints or in the annualized rate of SLE flares.

Among the subpopulation of patients with a high baseline interferon gene signature score (301 of 362 patients; $83.1 \%$ ), $48.0 \%$ of those treated with anifrolumab had a BICLA response, compared with $30.7 \%$ who received placebo; among patients with a low interferon gene signature, BICLA response occurred in $46.7 \%$ and $35.5 \%$, respectively.

Together, the phase III trials provide encouraging evidence of the efficacy of anifrolumab in SLE.

"The role of anifrolumab in the treatment paradigm should ideally be tested in treatment strategy trials, which we hope will happen soon," says Morand.

"In the case of any new drug for SLE, extensive real-world experience will be needed to gain a sense of the type of patients and clinical manifestations that are most responsive to the drug, as well as timing of administration and choice of concurrent or sequential other agents," Crow comments, adding that targeting of any one molecular pathway should not be expected to achieve satisfactory clinical improvement in many patients with SLE. "It is likely that strategically targeting several pathways will ultimately be required to achieve impressive clinical outcomes in patients with active SLE," she concludes.

\section{Sarah Onuora}

ORIGINAL ARTICLE Morand, E. F. et al. Trial of anifrolumab in active systemic lupus erythematosus. N. Engl.J. Med. 382, 211-221 (2020) RELATED ARTICLES Murphy, G. \& Isenberg, D. A. New therapies for systemic lupus erythematosus - past imperfect, future tense. Nat. Rev. Rheumatol. 15, 403-412 (2019) | Wampler Muskardin, T. \& Niewold, T. B. Type I interferon in rheumatic diseases. Nat. Rev. Rheumatol. 14, 214-228 (2018) 